\section{MS37-P12 Reticular Synthesis and \\ Structural Trends of Novel Cd(II) \\ Coordination Polymers Based on N-donor \\ Bridging Ligand \\ Mansoureh Zahedi ${ }^{1,2}$, Ulli Englert ${ }^{1}$} Landoltweg 1, 52074 Aachen, Germany

2. Department of inorganic chemistry,Faculty of chemistry, Tabriz University, Tabriz , Iran

\section{email: mzahedi83@gmail.com}

The rational design and syntheses of coordination polymers have attracted great attention from chemists not only because of their intriguing structural diversities and aesthetical topologies but also due to their interesting properties and potential applications as functional materials in gas storage/separation, heterogeneous catalysis, magnetism, photochemistry [1-4]. since the bulk properties of these materials are closely related to their structures, it is important to understand the factors such as coordination geometries of metal ions and the resulting secondary inorganic building blocks, spatial arrangements of organic building blocks, interpenetration, anions and solvents, which control their topologies. Taking the above discussion into account, in this study, we have systematically investigated construction, structural and property feature comparison of some new coordination polymers composed of a long and rigid $\mathrm{N}$-donor, bipyridine, linker ligand $(\mathrm{L})$ towards $\mathrm{CdX},(\mathrm{X}=$ $\left.\mathrm{CH}_{3} \mathrm{COO}^{-}, \mathrm{I}^{-}, \ldots\right)$. The $\mathrm{X}$-ray crystallography results display considerable structural differences in $\mathrm{Cd}$ (II) geometry. It includes one-dimensional ladder like polymeric chains with seven coordination numbers for $\mathrm{Cd}$ (II) metal center in the present of acetate anion, meanwhile, the tetrahedral geometry with zigzag chains have been seen with Iodide one.

\section{References:}

[1] J.L.C. Rowsell, O.M. Yaghi, Micropor. Mesopor. Mater. 2004,73, 3.

[2] Y. Ma, L. Zhang, G. Peng, C. Zhao, R. Dong, C. Yang and H. Deng CrystEngComm, 2014,16, 667-683.

[3] R. Patra, H. Titi and I. Goldberg, CrystEngComm, 2013, 15, 7257-7266.

[4] P. Wang, R. Fan, X. Liu, L. Wang, Y. Yang, W. Cao, B. Yang, W. Hasi, Q. Su and Y. Mu CrystEngComm, 2013,15, 1931-1949.

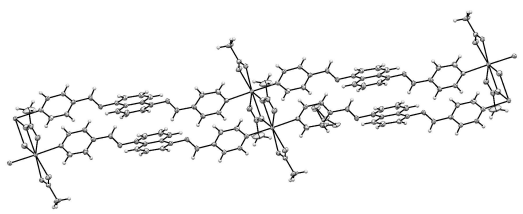

Figure 1. One dimensional structure of $\left[\mathrm{Cd}(\mathrm{L})(\mathrm{OAc})_{2}\right]$

Keywords: Coordination polymer, Bridging Ligand, Cadmium salts, X-ray Crystallogarphy

\section{MS37-P13 A Flexible Interpenetrated pcu Coordination Network Formed by Mixed Ligands \\ Mohana Shivanna ${ }^{1}$, Dr.QingYuan Yang ${ }^{2}$, Prof. Michael J Zaworotko ${ }^{3}$}

1. Mr. Mohana Shivanna, Doctor of philosophy, crystal engineering resrach group at University of Limerick, Ireland

2. Dr.QingYuan Yang, Post doctoral student, crystal engineering research group at university of limerick

3. Prof. Michael J. Zaworotko, Crystal engineering research group, University of Limerick

email: mohana.shivanna@ul.ie

Abstract: Metal-organic materials (MOMs), also known as porous coordination polymers (PCPs) or metal-organic frameworks (MOFs), are receiving attention thanks to their amenability to design and properties $[1,3]$. The pore size and chemistry of MOMs makes them excellent candidates for applications in storage, separation, sensing, and catalysis [2]. Flexible MOMs can exhibit behavior such as swelling, stepwise uptake, gate-opening and breathing which can be induced by stimuli such as solvent, pressure, heat and light $[4,5]$

Here, we report a two, three-fold interpenetrated pcu network with general formula [Zn4(L1)4(L2)2].DMF (L1=1,4-bis(4-carboxyphenyl)benzene; $\mathrm{L} 2=1,4$-bis

(4-pyridylbenzene), axially coordinated zinc "paddle-wheels", that exhibits dynamic structural transformations induced by guest incorporation and removal. X-ray structures highlight the highly flexible nature of the framework and reveal that phase transformations involve the movement or rotation of the biphenylene dicarboxylate ligands. The coordination geometry of a zinc paddle-wheel unit is considerably changed without bond breakage.

\section{References:}

1 Kitagawa, S. et al., Chem. Soc. Rev. 2005, 34, 109

2 Nugent, P. et al., Nature. 2013, 495, 80

3 Sakata, Y. et al., science . 2013, 339, 193

4 Schneemann, A. et al., Chem. Soc. Rev. 2014, 43, 6062

5 Mason, A. J. et al., Nature. 2015, 527, 357

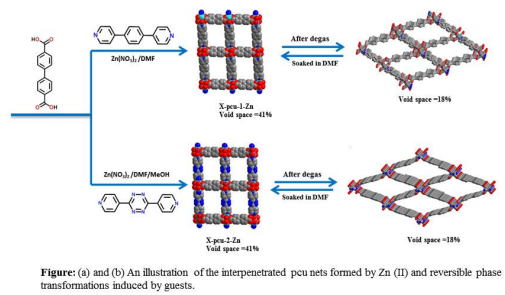

Figure 1. (a) and (b) An illustration of the interpenetrated pcu nets formed by $\mathrm{Zn}$ (II) and reversible phase transformations induced by guests. 